\title{
Solving MCDM problems based on combination of PACMAN and LINMAP
}

\author{
Mojtaba Kashef ${ }^{1}$ । Hossein Safari ${ }^{1}$ (D) । Meysam Maleki ${ }^{2}$ (D) । Virgilio Cruz-Machado ${ }^{2}$ (D)
}

${ }^{1}$ Department of Industrial Management, Faculty of Management, University of Tehran, Tehran, Iran

${ }^{2}$ UNIDEMI, Department of Industrial and Mechanical Engineering, Faculty of Science and Technology, Universidade Nova de Lisboa, Lisbon, Lisboa, Portugal

\section{Correspondence}

Meysam Maleki, UNIDEMI, Department of Industrial and Mechanical Engineering, Faculty of Science and Technology, Universidade Nova de Lisboa, Lisbon 2829-516, Lisboa, Portugal.

Email: maleki@fct.unl.pt

\begin{abstract}
Multicriteria decision-making approaches are receiving more and more attention with the increase of expectations from decision makers in variety of fields. The growth in applying such approaches has led to identifying their strengths as well as their shortcomings. Passive and active compensability multicriteria analysis (PACMAN) is one of the frequently used approaches which has the capability to consider compensation in describing intercriteria relations in multicriteria decision-making problems. This methodology is well formed and rationally structured in the first two phases, in which the problem is formulated and the decisive indices are obtained. However, it has some shortcomings in the idea of concluding the process of solution in its last phase. In the current study, we review the methodology, discuss its possible shortcomings, and propose an approach based on the combination of PACMAN and linear programming technique for multidimensional analysis of preference (LINMAP). The proposed approach which is taken to evaluate the PACMAN and LINMAP methodologies can help researchers and decision makers who seek an accurate perspective to evaluate a multicriteria decision-making methodology.
\end{abstract}

\section{KEYWORDS}

LINMAP, MCDM, PACMAN, ranking

\section{1 | INTRODUCTION}

Given the considerable amount of real-world problems with multiple criteria in their decision-making procedure, a massive body of research has been and is being done to present efficient, realistic, and easy-touse multicriteria decision-making (MCDM) methodologies. According to Matarazzo (1986), multicriteria analysis has helped authors taking a more realistic perspective towards decision-making problems. These studies have led scholars to develop approaches through exploiting combinations of published methodologies, notions, ideas, and sometimes through employing novel innovative notions and approaches (Chen, 2012; Gilliams, Raymaekers, Muys, \& Orshoven, 2005; Olson, 2001; Opricovic \& Tzeng, 2004; Reeves \& Gonzalez, 1989). The major differences between methodologies are in the way they formulate the problem: the type and number of inputs (representing the data required to employ the methodology), the idea of transforming inputs to outputs, and simplicity of outputs.
Opricovic and Tzeng (2004) describe the process of MCDM in six steps; in the first three steps, criteria and alternatives are determined; thereafter, alternatives are ranked with respect to criteria. Afterwards, the decision maker (DM) employs a normative multicriteria analysis. Because the cornerstone of the model is formed on the basis of this analysis, this step may be the most decisive step of the process, determining the type and number of data either required for the input or presented as the output. Table 1 sorts some of the frequently used MCDM methodologies into different classes based on the following perspectives: objectives, type of input data, results, quality, and output data.

Some of methodologies require few and general data. Thereupon, they could neglect some of the vital elements of the real problem. In particular, despite the fact that intercriteria relations are a decisive part of a MCDM problem, some methodologies such as AHP (Analytic Hierarchy Process; Saaty, 1980), TOPSIS (Technique for Order Performance by Similarity to Ideal Solution; Hwang \& Yoon, 1981), and VIKOR (Vlsekriterijumskaoptimizacija I Kompromisnoresenje in serbian; 
TABLE 1 Classifications of multicriteria decision-making methodologies

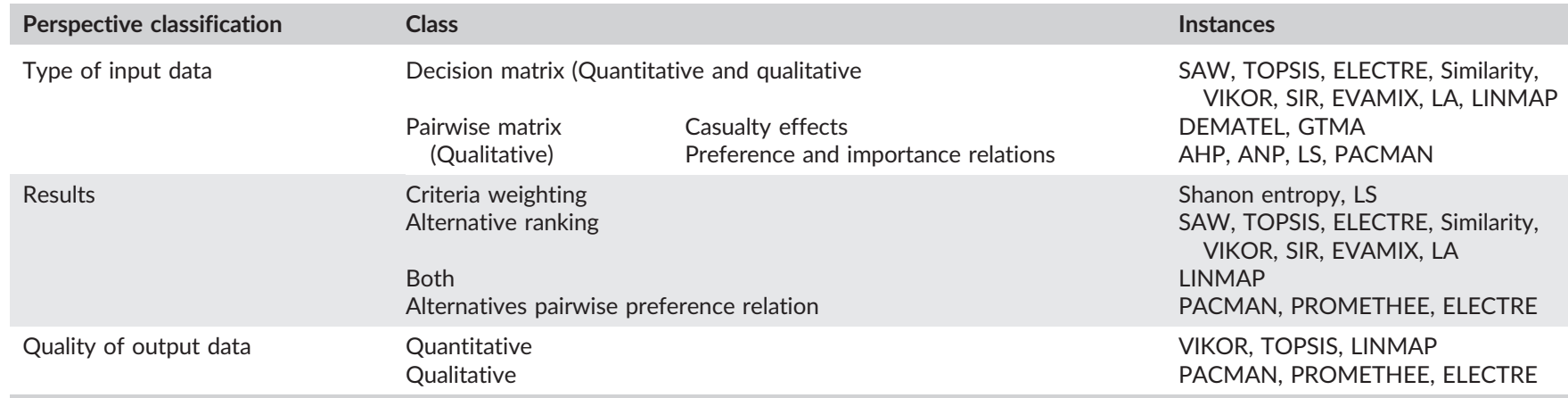

Opricovic, 1998) over simplify them to make their technique simpler (Afful-Dadzie, Nabareseh, Oplatková, \& Klímek, 2016; Baležentis \& Baležentis, 2014; Omar \& Fayek, 2016). These are usually basic methodologies which are easy to understand and employ. They model decision priorities by asking experts to express their opinions in the form of importance weights that usually do not have a clear economic significance (Opricovic \& Tzeng, 2004; Safari, Faraji, \& Majidian, 2016). Thus, more complex methodologies are often required to include such relations to model DMs' preferences in tackling problems (Zopounidis \& Pardalos, 2010). It has to be noted in the modelling procedure that methodologies are different in terms of reflecting the intercriteria relations to define the problem. For instance, DEMATEL, a methodology first generated by Batelle Memorial Institute of Geneava between 1972 and 1976 (Lin \& Tzeng, 2009), is a methodology which takes into account the intercriteria relations with the notion of influence. That is, it demands DMs to express their thoughts in terms of magnitude of impact each criterion has on every other criterion. These impacts finally determine the weight of the criterion.

Another example is the passive and active compensability multicriteria analysis (PACMAN) methodology (Brauers, 1998; Giarlotta, 1998) in which the notion of compensation allows one to determine an appropriate notion of weight for each criterion. In fact, in this approach, criteria can interact in a form that a negative difference of a specific criterion on a specific alternative can be compensated by a positive difference over another criterion for the same alternative. These notions and approaches in different methodologies all aim to formulate the problem, meeting the DM's constraints and priorities, in a way which is closer to what it is in real conditions (Rao, 2007).

Presentation of numerous researches in the field of MCDM has created a massive body of novel notions and methodologies. Although these research works contribute to the field in terms of improving the decision-making situation, they display their weaknesses as well. These weaknesses have to be properly addressed. Therefore, we study and analyse the proposed notions and methodologies to find their strengths and shortcomings and suggest ideas to improve strengths and cover shortcomings. It will eventually contribute to make methodologies easier to use, formulate the problem better, and present more tangible results.

The current research intends to perform a practical evaluation of a recent methodology, linear PACMAN, and review it in order to detect its strengths and weaknesses. Moreover, considering the weak points and features to improve, an idea of combining the linear PACMAN methodology with linear programming technique for multidimensional analysis of preference (LINMAP) is presented.

It is worth mentioning here that the aim of this research is to emphasize on the importance of the intercriteria relations, scrutinize PACMAN as a methodology that takes these relations into account, and propose solutions to cover its potential shortcomings so that a wider body of researchers benefit from its strengths.

The remainder of the paper is organized as follows. In Second 2, PACMAN and LINMAP are described, and possible weaknesses of PACMAN are discussed. In Section 3, our new approach, which combines these two methodologies in an effective way, is presented, along with an illustrative example. Section 3 also is dedicated to discussion, and the Section 4 concludes the paper.

\section{2 | MATERIAL AND METHOD}

One of the major concerns in MCDM methodologies is weather and how to consider intercriteria relations (Giarlotta, 1998). General MCDM methodologies such as AHP and TOPSIS neglect these interrelations wholly and solve the problem with the assumption that criteria are independent. Despite their shortcomings, these methodologies have been used in various applications as basis to propose new ideas and tools (Calabrese, Costa, \& Menichini, 2013; Ertuğrul \& Karakaşoğlu, 2009; Gupta \& Mohanty, 2016; Krohling \& Campanharo, 2011; Lee, Jun, \& Chung, 2013; Ordoobadi, 2010; Sadat, Safari, Sadabadi, \& Khanmohammadi, 2016; Safari \& Soufi, 2015; Rouhani, Ghazanfari, \& Jafari, 2012; Wang, Cheng, \& Huang, 2009; Zheng, Zhu, Tian, Chen, \& Sun, 2012). However, given the fact that the existence of intercriteria relations is undeniable, these methodologies possess structural weakness in formulating the problem as it really is. Thus, researchers have proposed techniques and methodologies to involve the intercriteria relations in portraying the problem. As mentioned earlier, one of the methodologies which is able to consider the intercriteria relations is PACMAN. It proposes the concept of "compensation" to explain the way criteria interrelate. In the following section, the methodology of linear PACMAN-which is a simpler form of PACMAN, useful to handle practical cases-is described. 


\section{1 | Linear PACMAN}

\subsection{1 | Methodology}

Based on a notion of compensability, Giarlotta (1998) proposed a new approach in multiple criteria decision aiding. He suggested that the intercriteria relations for two given criteria could be expressed by means of the possibility of an improvement in one, convincing the DM to neglect a deterioration in the other. Nowadays, the methodology is considered as one of the so-called outranking methodologies, whereas the well-known ELECTRE methodologies are considered as older outranking methodologies. Speaking of the difficulties that later led to development of ELECTRE methodologies, Roy and Vanderpooten (1996), the leading author in the field, report that an idea of compensation was first suggested in 1965 in a method named MARSAN:

... compensation phenomena could lead to assigning a better score to an alternative $a$, which was preferred to an alternative $b$ with respect to many criteria but which was much worse than $b$ for one criterion.

PACMAN suggests the intercriteria relations be discovered through interviews with DM. The approach is thus regarded a DM-oriented one. A peculiar feature of the notion of compensability is the idea of discrimination between the compensating criterion from the compensated one in a given compensation relation. This feature has caused the approach to be nonasymmetric. Namely, the index which demonstrates the preference degree of one alternative over another does not necessarily have either an asymmetric relation or any kind of other relation with the index showing the contrary preference degree.

A general PACMAN methodology supposes that criteria interrelate through different, free, and unrestricted functions. It also allows the DM to choose the intercriteria compensability relations, the so-called auxiliary and aggregation functions, and the sensitivity thresholds, whatever that best fits the real problem in the real world. Later, however, Angilella and Giarlotta (2009) introduced restricted formulations of PACMAN in order to control the so-called "excessive flexibility" laid in the approach. To do so, first, they studied different applications of PACMAN, looking for more widely applied functions. Thereupon, they proposed a new, predefined, and restricted form of PACMAN-the lexicographic implementation of PACMAN.

Angilella, Giarlotta, and Lamantia (2010) presented another predefined form of PACMAN, which is less complicated in intercriteria relations, and is well suited to MCDM problems. Having the same philosophy as the general PACMAN and being simple to understand, the rest of this paper addresses the methodology of linear PACMAN. All the possible modifications and suggestions presented in this paper can be applied to all forms of PACMAN. Further information about the general PACMAN methodology and its phases can be found in Angilella and Giarlotta (2009) and Giarlotta (1998, 2001) discussing the linear implementation of PACMAN.

The sequential steps of linear PACMAN are as follows. Readers are recommended to see Angilella et al. (2010) for a more detailed description of linear PACMAN.

1. Gathering the DM opinions on compensability within each ordered pair of criteria. ${ }^{1}$
2. Determining $\lambda_{i j^{(0)}}$ and $\lambda_{i j^{(1)}}$ for each ordered pair of criteria. ${ }^{2}$

3. Constructing the compensability function for each ordered pair of criteria based on the following expression:

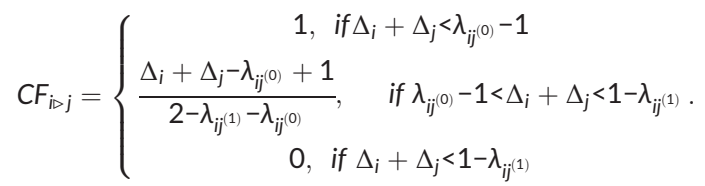

$\Delta_{i}$ and $\Delta_{j}$ are variables.

4. Computing the active and passive elementary indices $\dot{\pi}_{i \triangleright j}^{+}\left(a_{p}, a_{q}\right)$ and $\dot{\pi}_{j>i}^{-}\left(a_{p}, a_{q}\right)$ in ordinal manner for each ordered pair of criteria and each ordered pair of alternatives based on the following:

$$
\begin{gathered}
\dot{\pi}_{i \triangleright j}^{+}\left(a_{p}, a_{q}\right)=\left\{\begin{array}{c}
1, \Delta_{i}>0 \text { and } \Delta_{j} \geq 0 \\
C F_{i \triangleright j}\left(-\Delta_{i},-\Delta_{j}\right), \Delta_{i}>0 \text { and } \Delta_{j}<0 . \\
0, \Delta_{j} \leq 0
\end{array}\right. \\
\dot{\pi}_{j \triangleright i}^{-}\left(a_{p}, a_{q}\right)=\left\{\begin{array}{c}
1, \Delta_{i}>0 \text { and } \Delta_{j} \geq 0 \\
1-C F_{i \triangleright j}\left(-\Delta_{i},-\Delta_{j}\right), \Delta_{i}>0 \text { and } \Delta_{j}<0 . \\
0, \Delta_{j} \leq 0
\end{array}\right.
\end{gathered}
$$

The active and passive elementary indices in the cardinal manner are computed using these relations:

$$
\begin{aligned}
& \ddot{\pi}_{i \triangleright j}^{+}\left(a_{p}, a_{q}\right)=\Delta_{i}\left(a_{p}, a_{q}\right) \dot{\pi}_{i \triangleright j}^{+}\left(a_{p}, a_{q}\right), \\
& \ddot{\pi}_{j \triangleright i}^{-}\left(a_{p}, a_{q}\right)=\Delta_{j}\left(a_{p}, a_{q}\right) \dot{\pi}_{j \triangleright i}^{-}\left(a_{p}, a_{q}\right),
\end{aligned}
$$

where

$$
\Delta_{i}\left(a_{p}, a_{q}\right)=\frac{a_{p i}-a_{q i}}{\beta_{i}-\alpha_{i}} .
$$

5. Aggregating the elementary indices to the active and passive partial indices, $\pi_{i}^{+}\left(a_{p}, a_{q}\right)$ and $\pi_{i}^{-}\left(a_{p}, a_{q}\right)$ according to the following:

$$
\begin{aligned}
& \pi_{i}^{+}\left(a_{p}, a_{q}\right)=\varphi_{i}^{+}\left(\dot{\pi}_{i \triangleright k}^{+}\left(a_{p}, a_{q}\right): k \in J \backslash\{i\},\right. \\
& \pi_{i}^{-}\left(a_{p}, a_{q}\right)=\varphi_{i}^{-}\left(\dot{\pi}_{h \triangleright i}^{-}\left(a_{p}, a_{q}\right): \mathrm{h} \in J \backslash\{i\} .\right.
\end{aligned}
$$

In case, the elementary indices are computed in an ordinal manner.

and

$$
\begin{aligned}
& \pi_{i}^{+}\left(a_{p}, a_{q}\right)=\varphi_{i}^{+}\left(\ddot{\pi}_{i \triangleright k}^{+}\left(a_{p}, a_{q}\right)\right): \mathrm{k} \in J \backslash\{i\}, \\
& \pi_{i}^{-}\left(a_{p}, a_{q}\right)=\varphi_{i}^{-}\left(\ddot{\pi}_{h \triangleright i}^{-}\left(a_{p}, a_{q}\right)\right): \mathrm{h} \in J \backslash\{i\} .
\end{aligned}
$$

In case, the elementary indices are computed in a cardinal manner.

The functions $\varphi_{i}^{+}$and $\varphi_{i}^{-}$are both simple arithmetic mean.

6. Aggregating the partial indices $\pi_{i}^{+}\left(a_{p}, a_{q}\right)$ and $\pi_{i}^{-}\left(a_{p}, a_{q}\right)$ obtained in the last step to the active and passive global indices for each ordered pair of alternatives

$$
\pi^{+}\left(a_{p}, a_{q}\right)=\varphi^{+}\left(\pi_{i}^{+}\left(a_{p}, a_{q}\right)\right): i \in J,
$$




$$
\pi^{-}\left(a_{p}, a_{q}\right)=\varphi^{-}\left(\pi_{i}^{-}\left(a_{p}, a_{q}\right)\right): i \epsilon J
$$

7. Obtaining the net global index, $\pi\left(a_{p}, a_{q}\right)$, for each ordered pair of alternatives as follows:

$$
\pi\left(a_{p}, a_{q}\right)=\pi^{+}\left(a_{p}, a_{q}\right)-\pi^{-}\left(a_{q}, a_{p}\right),
$$

in which $\pi^{-}\left(a_{q}, a_{p}\right)$ is the passive global index of alternatives $a_{q}$ and $a_{p}$ and $\pi^{+}\left(a_{p}, a_{q}\right)$ is the active index of alternatives $a_{p}$ and $a_{q}$.

8. Choosing the sensitivity threshold, $\varepsilon$, based on which global indices are transformed to binary preference indices, $\varphi_{a_{p}, a_{q}}$. This is done by the DM.

9. Obtaining the binary preference indices, $\varphi_{a_{\mathrm{p}}, \mathrm{a}_{\mathrm{q}}}$, within each ordered pair of alternatives according to the following:

$$
\varphi_{a_{p}, a_{q}}=\left\{\begin{array}{cc}
1, & \pi\left(a_{p}, a_{q}\right) \epsilon(\varepsilon, 1] \\
0, & \pi\left(a_{p}, a_{q}\right) \epsilon[-\varepsilon, \varepsilon] \\
-1, & \pi\left(a_{p}, a_{q}\right) \epsilon[-1,-\varepsilon)
\end{array} .\right.
$$

10. The preference relations are now as follows:

a) Strong preference:

$$
a_{p} P a_{q} \leftrightarrow \varphi_{a_{p}, a_{s}}=1, \varphi_{a_{q}, a_{p}}=-1 .
$$

b) Weak preference

$$
\begin{aligned}
& a_{p} Q a_{q} \leftrightarrow \varphi_{a_{p}, a_{s}}=1, \\
& \varphi_{a_{q}, a_{p}}=0 \text { or } \varphi_{a_{p}, a_{q}}=0, \varphi_{a_{q}, a_{p}}=-1 .
\end{aligned}
$$

c) Indifferences

$$
a_{p} l a_{q} \leftrightarrow \varphi_{a_{p}, a_{\mathrm{s}}}=1, \quad \varphi_{a_{q}, a_{p}}=1, \operatorname{or} \varphi_{a_{p}, a_{q}}=0, \varphi_{a_{q}, a_{p}}=0 .
$$

d) Incomparable

$$
a_{p} R a_{q} \leftrightarrow \varphi_{a_{p}, a_{s}}=-1, \varphi_{a_{q}, a_{p}}=-1
$$

Possible shortcomings of PACMAN methodology is put forward in the next subsection.

\subsection{2 | Possible shortcomings}

Alongside the strong, realistic formulation of the linear PACMAN methodology, there are points of improvement as well. The content of this section presents points that can prevent DM's from taking advantage of the well-structured notion of compensability.

In Step 8 of linear PACMAN, the DM is required to determine a threshold, $\varepsilon \in[0,1]$, based on which global indices will be transformed to binary preference indices which in turn determine the eventual preference relations between alternatives. Many DMs may feel more convenient if they are relieved from deciding such a rather complicated, yet decisive, parameter merely on their own.

Matarazzo (1986) insists adequate interpretation of the results acquired is one of the two important properties of the "operative aspect" of a multicriteria analysis method. As already seen, the final result is a set of pairwise comparisons. These predefined comparisons are the general product of outranking methodologies, same as described in Roy (1990). To develop a ranking out of PACMAN results, the DM has to take into consideration a number of $m(m-1) / 2$ pairwise comparisons which is often not a convenient number. For instance, with 10 alternatives to rank, there are 45 comparisons to be considered at one time. On the other hand, the methodology includes an "incomparable", R, relation (apart from the "indifference", I, relation) which leaves the relation of some alternatives with no preference. Thereupon, there may be problems in which even after a scrutiny of $m(m-1) / 2$ pairwise comparisons, not all the alternatives are ranked in a total preorder. This could rub those DM's who tend to have a total preorder of alternatives from the advantages of linear PACMAN's notion of compensability. In addition, with qualitative comparisons among alternatives, DM's who look for a chance to analyse tangible differences among alternatives, are not served at all.

\section{2 | LINMAP}

\subsection{1 | Methodology}

Srinivasan and Shocker (1973) introduced LINMAP methodology, a ranking methodology employing two inputs from the DM: a decision matrix and a set of pairs of alternatives, $S$, in which existence of a pair $(K, L)$ indicates $D M ' s$ total preference of alternative $K$ over alternative L. The methodology afterwards, constructs a linear programming. Solving the linear programming, weights of criteria as well as rates of an imaginary ideal solution against each criterion are obtained. Every alternative is then compared with others based on its Euclidean distance from the ideal solution.

LINMAP has been applied to MCDM problems in different fields (Bereketli, Erol Genevois, Esra Albayrak, \& Ozyol, 2011; Zhang \& Lu, 2006), and some of the researchers have suggested expansions and modifications on it (Li, 2008; Mullet \& Karson, 1986; Xia, Li, Zhou, \& Wang, 2006).

A step-by-step implementation of LINMAP contains the following. More details are found in Srinivasan and Shocker (1973).

1. Pooling the DM's ideas on the preference relations between alternatives. This step develops the set $S=\{(K, L)\}, K, L \in A$.

2. Solving the following linear mathematical programming:

$$
\begin{aligned}
& \text { Min } Z=\sum_{K, L E S} \varphi K, L \\
& \sum_{j=1}^{n} w_{j}\left(r_{l j}^{2}-r_{k j}^{2}\right)-2 \sum_{j=1}^{n} v_{j}\left(r_{l j}-r_{k j}\right)+\varphi_{K, L} \geq 0 \\
& \sum_{j=1}^{n} w_{j} \sum\left(r_{L j}^{2}-r_{K j}^{2}\right)-2 \sum_{j=1}^{n} v_{j} \sum\left(r_{L j}-r_{K j}\right) \geq h \\
& \varphi_{K, L} \geq 0, w_{j} \geq 0 .
\end{aligned}
$$

3. Computing the ideal solution rates, $r_{j}^{*}$ by applying the following equation:

$$
v_{j}=w_{j} \times r_{j}^{*} .
$$

4. Obtaining the Euclidean distances of different alternatives from the ideal solution based on the following:

$$
d_{K}=\sqrt{\sum_{j \in J\left\{j^{\prime}\right\}} w_{j}\left(r_{K j}-r_{j}^{*}\right)^{2}-2 \sum_{j^{\prime}} v_{j^{\prime}}\left(r_{K j^{\prime}}\right)},
$$


where

$$
j^{\prime}=\left\{j \in J \mid r_{j}^{*}= \pm \infty\right\}
$$

5. Ranking the alternatives; the less its distance from the ideal solution, the better the alternative.

\section{3 | THE PROPOSED APPROACH}

\section{1 | Theory}

With the net global indices obtained in PACMAN's Step 7, the DA will have a set of preference relations for every possible ordered pair of alternatives. Putting these together as a set $\mathrm{S}$, one will be able to analyse them, applying LINMAP, to acquire a quantitative score for every alternative. The DM on the other hand, will not be required to determine any threshold.

The proposed approach suggests a matrix $S=\left\{\pi\left(a_{r}, a_{s}\right)\right\}, r, s \in A$ of continuous indices of preference relations as the input to LINMAP. Simultaneous existence of both pairs $\left(a_{r}, a_{s}\right)$ and $\left(a_{s}, a_{r}\right)$ in set $S$ is not impossible. With such a combination, the DA is able to conclude to a total preorder of alternatives, benefiting from possible difference analysis.

The proposed approach, after PACMAN's Step 7, continues as follows:

1. Collecting the indices $\pi\left(a_{p}, a_{q}\right)$ in the matrix $S$ for all the $m(m-1)$ ordered pairs of alternatives.

2. Solving the following linear mathematical programming:

$$
\begin{aligned}
& \text { Min } Z=\sum_{p, q \in A, p \neq q} \varphi_{p, q} \\
& \pi\left(a_{p}, a_{q}\right) \sum_{j=1}^{n} w_{j}\left(r_{q j}^{2}-r_{p j}^{2}\right)-2 \pi\left(a_{p}, a_{q}\right) \sum_{j=1}^{n} v_{j}\left(r_{q j}-r_{p j}\right)+\varphi_{p, q} \geq 0 \\
& \sum_{j=1}^{n} w_{j} \sum \pi\left(a_{p}, a_{q}\right)\left(r_{q j}^{2}-r_{p j}^{2}\right)-2 \sum_{j=1}^{n} v_{j} \sum \pi\left(a_{p}, a_{q}\right)\left(r_{q j}-r_{p j}\right) \geq h \\
& \varphi_{p, q} \geq 0, w_{j} \geq 0 .
\end{aligned}
$$

3. Computing the ideal solution rates, $r_{j}^{*}$ by applying the following equation:

$$
v_{j}=w_{j} \times r_{j}^{*}
$$

4. Obtaining the Euclidean distances of different alternatives from the ideal solution based on the following:

$$
\begin{aligned}
d_{p} & =\sqrt{\sum_{j \in J\left\{j^{\prime}\right\}} w_{j}\left(r_{p j}-r_{j}^{*}\right)^{2}-2 \sum_{j^{\prime}} v_{j^{\prime}}\left(r_{p j^{\prime}}\right)} \text { where } J^{\prime} \\
& =\left\{j \in J \mid r_{j}^{*}= \pm \infty\right\} .
\end{aligned}
$$

5. Ranking the alternatives; the less the distance from the ideal solution, the better the alternative.

\section{2 | An example}

A numerical example is presented in this section to clarify the proposed approach. Angilella et al. (2010) presented an illustrative example which is an MCDM problem of ranking three cars as alternatives based on a set of three criteria as price, maximum speed, and fuel consumption. In order to make comparison easier, we solve the same example employing the proposed approach and discuss the results. Because the discussion of authors in the above work encompasses a part in which the cardinal manner is urged to be more appropriate in dealing with decision problems due to some shortcomings of the ordinal implementation (Angilella et al., 2010), we consider the cardinal implementation results as the representative of PACMAN methodology.

It has to be noticed that the indices of the first phase of the proposed approach which are computed using PACMAN are imported directly from the work by Angilella et al. (2010) and are not repeated here to avoid redundancy.

Suppose the decision matrix is like the following (Table 2):

According to step-by-step implementation of the proposed approach, the first seven steps are shared with the first two phases of PACMAN. Thereafter, inserting the results of PACMAN, the matrix $S$ will be developed (Table 3).

Subsequently, having the decision matrix and net global indices, it is possible to construct the following linear mathematical programming based on expression ((31)):

$$
\operatorname{Min} Z=\varphi_{a, b}+\varphi_{a, c}+\varphi_{b, a}+\varphi_{b, c}+\varphi_{c, a}+\varphi_{c, b}
$$

Subject to

(constraint for the ordered pair $(a, b)$ )

$$
\begin{aligned}
0.293 & {\left[\left(w_{1}(0)^{2}-(-1)^{2}\right)+\left(w_{2}(0)^{2}-(1)^{2}\right)+\left(w_{3}(1)^{2}-(0)^{2}\right)\right] } \\
& -2(0.293)\left[\left(v _ { 1 } \left(0-(-1)+\left(v_{2}(0-1)\right)+\left(v_{1}(1-0)\right]+\varphi_{c, a}\right.\right.\right. \\
& =0.293 w_{1}+0.293 w_{2}-0.293 w_{3}+0.586 v_{1}-0.586 v_{2} \\
& +0.586 v_{3}+\varphi_{a, b} .
\end{aligned}
$$

For each of the remaining ordered pairs too, there is a constraint like the above which is formed likewise. Apart from them, other constraints are as follows:

$$
w_{1}+w_{2}+w_{3}=1
$$

$$
(-0.61) w_{1}+0.56 w_{2}-0.945 w_{3}+0.68 v_{1}-0.67 v_{2}+1.16 v_{3} \geq 1
$$

TABLE 2 The decision matrix (Angilella et al., 2010)

\begin{tabular}{llll} 
& Price & Maximum speed & Fuel consumption \\
\hline Car 1 & 16,000 & 180 & 15 \\
\hline Car 2 & 15,000 & 160 & 16 \\
\hline Car 3 & 12,000 & 130 & 25 \\
\hline
\end{tabular}

TABLE 3 The matrix S demonstrating linear PACMAN final indices (Angilella et al., 2010)

$\begin{array}{cccc} & \text { a } & \text { b } & c \\ \text { a } & - & -0.012 & -0.417 \\ \text { b } & -0.023 & - & -0.459 \\ \text { c } & 0.293 & 0.365 & -\end{array}$


TABLE 4 Solution of the linear programming included in the proposed methodology

\begin{tabular}{lllr}
$\mathbf{J}$ & $\mathbf{w}^{* \mathbf{j}}$ & $\mathbf{v}^{* \mathbf{j}}$ & $\mathbf{r}^{* \mathbf{j}}$ \\
1 & 0.00 & 2.17 & $+\infty$ \\
2 & 0.00 & 1.34 & $+\infty$ \\
3 & 1.00 & 0.00 & 0 \\
\hline
\end{tabular}

TABLE 5 Final results produced by the proposed methodology

$\begin{array}{lll}\text { Alternatives } & \text { d } & \text { Rank } \\ \text { a } & 1.285 & 2 \\ \text { b } & 1.285 & 3 \\ \text { c } & 1 & 1\end{array}$

TABLE 6 Sensitivity analysis of PACMAN final results with respect to changes in the parameter $\varepsilon$

\begin{tabular}{llll}
\hline Intervals for $\varepsilon$ & a and b status & $\mathbf{b}$ and $\mathbf{c}$ status & $\mathbf{a}$ and $\mathbf{c}$ status \\
\hline$[0.0000,0.0119]$ & $\mathrm{aRb}$ & $\mathrm{cPb}$ & $\mathrm{cPa}$ \\
{$[0.0120,0.0229]$} & $\mathrm{aQb}$ & $\mathrm{cPb}$ & $\mathrm{cPa}$ \\
{$[0.0230,0.2929]$} & $\mathrm{alb}$ & $\mathrm{cPb}$ & $\mathrm{cPa}$ \\
{$[0.2930,0.3649]$} & $\mathrm{alb}$ & $\mathrm{cPb}$ & $\mathrm{cQa}$ \\
{$[0.3650,0.4169]$} & $\mathrm{alb}$ & $\mathrm{cQb}$ & $\mathrm{cQa}$ \\
{$[0.4170,0.4589]$} & alb & $\mathrm{cQb}$ & $\mathrm{cla}$ \\
{$[0.4590,1.0000]$} & $\mathrm{alb}$ & $\mathrm{cQb}$ & $\mathrm{cla}$ \\
\hline
\end{tabular}

$$
\varphi_{p, q} \geq 0, w_{j} \geq 0
$$

The above linear programming problem is solved to obtain the final results.

\section{3 | Results and discussions}

Table 4 reveals the output of the linear programming.

Possessing $\mathrm{w}^{*} \mathrm{j}$ and $\mathrm{r}^{*} \mathrm{j}$ for all $\mathrm{j} \in \mathrm{J}$, the distances of alternatives can be obtained through formula (11). Table 5 presents final results.

Precise scrutiny in results indicates that there is a difference of very little magnitude $\left(2.22 \times 10^{-16}\right)$ between distances of alternatives $a$ and $b$ from the ideal alternative.

The sensitivity analysis of final results according to changes in $\varepsilon$ is calculated in Table 6. Cells with grey background highlight the change in final results with moving on to the next interval of $\varepsilon$. The table clarifies that even slight changes in the parameter $\varepsilon$ may change the status between two (or more) alternatives. Angilella et al. (2010) set the parameter at 0.05 which results in a strong preference of car cover cars $a$ and $b$ and indifference between cars $a$ and $b$ themselves.

Looking at results of the proposed approach, the first significant point is that this approach leads to the same results not with qualitative indices but with continuous indices. This gives the DA the opportunity to accurately analyse the differences (Table 7). Moreover, the proposed approach does not require the DM to set sensitive parameter of $\varepsilon$ and therefore the final result is unique.
TABLE 7 Difference in percentage of final indices of alternatives from the ideal solution

\begin{tabular}{ll} 
Alternatives & $\begin{array}{l}\text { Difference in final indices (distance from ideal } \\
\text { alternative) }\end{array}$ \\
\hline $\mathrm{a}$ and $\mathrm{b}$ & $\approx 0.00 \%$ \\
$\mathrm{~b}$ and $\mathrm{c}$ & $28.53 \%$ \\
$\mathrm{a}$ and $\mathrm{c}$ & $28.53 \%$ \\
\hline
\end{tabular}

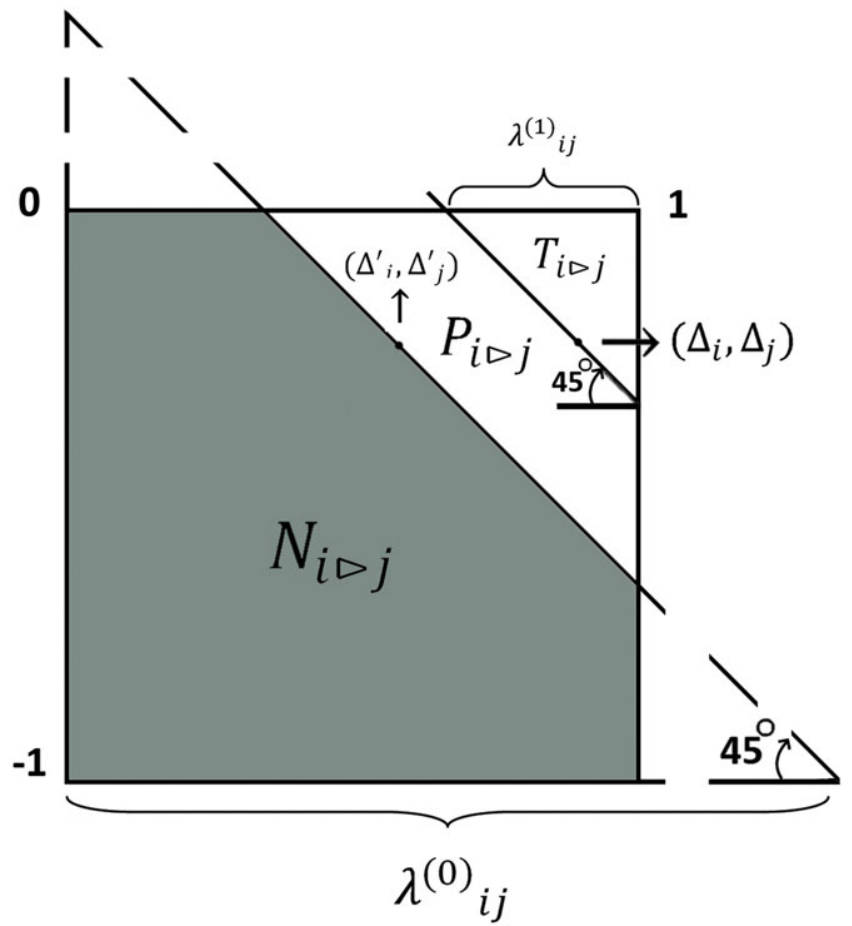

FIGURE 1 Schematic presentation of $\lambda_{\mathrm{ij}}\left({ }^{(0)}\right.$ and $\lambda_{\mathrm{ij}}(1)$

\section{4 | CONCLUSIONS}

In the current research, a novel approach is introduced combining two methodologies existing in the literature, namely, linear PACMAN and LINMAP. The idea of combining the two methodologies starts from shortcomings in the PACMAN methodology, mostly in the last phase where a fundamental system of preferences is established among alternatives, and also the compatibility of LINMAP inputs to receive and process the outputs of linear PACMAN methodology at the second phase. This combination covers some of LINMAP weaknesses as well.

More analysable indices, less parameters required from the DM, more consistency with the real decision problem conditions, and uniqueness and certainty of results are the major advantages of the proposed approach in comparison with the two individual methodologies, specially the methodology of linear PACMAN which plays cornerstone role in the proposed approach.

\section{ENDNOTES}

${ }^{1}$ The DM is asked to answer four questions:a. What positive difference in criteria $\mathrm{i}, \Delta_{i}$, totally compensates for a negative difference of $\Delta_{j}$ ? For instance, for choosing a car, what positive difference in horse 
power totally compensates a $400 \$$ higher price?b. What smaller $\Delta_{i}$ still totally compensates for the same $\Delta_{j}$ ?c. What positive difference in criteria $\mathrm{i}, \Delta_{\mathrm{i}}^{\prime}$, does NOT AT ALL compensate for a negative difference of $\Delta_{j}^{\prime}$ ?d. What greater $\Delta_{i}^{\prime}$ still does NOT AT ALL compensate the same $\Delta_{j}^{\prime}$ ?

$2 \lambda_{\mathrm{ij}(0)}$ and $\lambda_{\mathrm{ij}(1)}$ are two non-negative parameters, satisfying the below conditions:

$$
\lambda_{i j}^{(0)}+\lambda_{i j}^{(1)} \leq 2 \text { if } \lambda_{i j}^{(0)}+\lambda_{i j}^{(1)}=2 \rightarrow \text { either } \lambda_{i j}^{(0)}=0 \text { or } \lambda_{i j}^{(1)}=0
$$

Figure 1 helps readers to understand how precisely $\lambda_{\mathrm{ij}}(0)$ and $\lambda_{\mathrm{ij}}{ }^{(1)}$ are obtained. $\left(\Delta_{\mathrm{i}}, \Delta_{\mathrm{j}}\right)$ and $\left(\Delta_{\mathrm{i}}^{\prime}, \Delta_{\mathrm{j}}^{\prime}\right)$ were determined in questions (b) and (d) above.

\section{ORCID}

Hossein Safari (D) http://orcid.org/0000-0001-9232-1319

Meysam Maleki (D) http://orcid.org/0000-0002-1111-0358

Virgilio Cruz-Machado (D) http://orcid.org/0000-0002-1165-4339

\section{REFERENCES}

Afful-Dadzie, E., Nabareseh, S., Oplatková, Z. K., \& Klímek, P. (2016). Model for assessing quality of online health information: A fuzzy VIKOR based method. Journal of Multi-Criteria Decision Analysis, 23(1-2), 49-62.

Angilella, S., \& Giarlotta, A. (2009). Implementations of PACMAN. European Journal of Operational Research, 194(2), 474-495.

Angilella, S., Giarlotta, A., \& Lamantia, F. (2010). A linear implementation of PACMAN. European Journal of Operational Research, 205(2), 401-411.

Baležentis, T., \& Baležentis, A. (2014). A survey on development and applications of the multi-criteria decision making method MULTIMOORA. Journal of Multi-Criteria Decision Analysis, 21(3-4), 209-222.

Bereketli, I., Erol Genevois, M., Esra Albayrak, Y., \& Ozyol, M. (2011). WEEE treatment strategies' evaluation using fuzzy LINMAP method. Expert Systems with Applications, 38(1), 71-79.

Brauers, W. K. (1998). A non-linear utility theory with multiple objectives. Journal of Multi-Criteria Decision Analysis, 7(4), 193-203.

Calabrese, A., Costa, R., \& Menichini, T. (2013). Using fuzzy AHP to manage intellectual capital assets: An application to the ICT service industry. Expert Systems with Applications, 40(9), 3747-3755.

Chen, T.-Y. (2012). Comparative analysis of SAW and TOPSIS based on interval-valued fuzzy sets: Discussions on score functions and weight constraints. Expert Systems with Applications, 39(2), 1848-1861.

Ertuğrul, ì., \& Karakaşoğlu, N. (2009). Performance evaluation of Turkish cement firms with fuzzy analytic hierarchy process and TOPSIS methods. Expert Systems with Applications, 36(1), 702-715.

Giarlotta, A. (1998). Passive and active compensability multi criteria analysis (PACMAN). Journal of Multi-Criteria Decision Analysis, 7(4), 204-216.

Giarlotta, A. (2001). Multicriteria compensability analysis. European Journal of Operational Research, 133(1), 190-209.

Gilliams, S., Raymaekers, D., Muys, B., \& Orshoven, J. V. (2005). Comparing multiple criteria decision methods to extend a geographical information system on afforestation. Computers and Electronics in Agriculture, 49(1), 142-158.

Gupta, M., \& Mohanty, B. K. (2016). Attribute partitioning in multiple attribute decision making problems for a decision with a purpose -A fuzzy approach. Journal of Multi-Criteria Decision Analysis, 23(3-4), 160-170.

Hwang, C. L., \& Yoon, K. (1981). Multiple attribute decision making: Methods and applications ( ed., Vol. 1981). New York: Springer.

Krohling, R. A., \& Campanharo, V. C. (2011). Fuzzy TOPSIS for group decision making: A case study for accidents with oil spill in the sea. Expert Systems with Applications, 38(4), 4190-4197.
Lee, G., Jun, K. S., \& Chung, E.-S. (2013). Robust spatial flood vulnerability assessment for Han River using fuzzy TOPSIS with a-level sets. Expert Systems with Applications.. in press

Li, D.-F. (2008). Extension of the LINMAP for multi attribute decision making under Atanassov's intuitionistic fuzzy environment. Fuzzy Optimization and Decision Making, 7(1), 17-34.

Lin, C. L., \& Tzeng, G. H. (2009). A value-created system of science (technology) park by using DEMATEL. Expert Systems with Applications, 36(6), 9683-9697.

Matarazzo, B. (1986). Multicriterion analysis of preferences by means of pairwise actions and criterion comparisons (MAPPACC). Applied Mathematics and Computation, 18(2), 119-141.

Mullet, G. M., \& Karson, M. J. (1986). Percentiles of LINMAP conjoint indices of fit for various orthogonal arrays: A simulation study. Journal of Marketing Research, 23, 286-290.

Olson, D. L. (2001). Comparison of three multicriteria methods to predict known outcomes. European Journal of Operational Research, 130(3), 576-587.

Omar, M. N., \& Fayek, A. R. (2016). A TOPSIS-based approach for prioritized aggregation in multi-criteria decision-making problems. Journal of Multi-Criteria Decision Analysis, 23(5-6), 197-209.

Opricovic, S. (1998). Multicriteria optimization of civil engineering systems. Faculty of Civil Engineering, Belgrade, 2(1), 5-21.

Opricovic, S., \& Tzeng, G. H. (2004). Compromise solution by MCDM methods: A comparative analysis of VIKOR and TOPSIS. European Journal of Operational Research, 156(2), 445-455.

Ordoobadi, S. M. (2010). Application of AHP and Taguchi loss functions in supply chain. Industrial Management \& Data Systems, 110(8), 1251-1269.

Rao, R. V. (2007). Decision making in the manufacturing environment: Using graph theory and fuzzy multiple attribute decision making methods. Springer Verlag.

Reeves, G. R., \& Gonzalez, J. J. (1989). A comparison of two interactive MCDM procedures. European Journal of Operational Research, 41(2), 203-209.

Rouhani, S., Ghazanfari, M., \& Jafari, M. (2012). Evaluation model of business intelligence for enterprise systems using fuzzy TOPSIS. Expert Systems with Applications, 39(3), 3764-3771.

Roy, B. (1990). The outranking approach and the foundations of ELECTRE methods. In Readings in multiple criteria decision aid. (pp. 155-183). Berlin, Heidelberg: Springer.

Roy, B., \& Vanderpooten, D. (1996). The European school of MCDA: Emergence, basic features and current works. Journal of Multi-Criteria Decision Analysis, 5(1), 22-38.

Saaty, T. L. (1980). The analytic hierarchy process: Planning, priority setting, resources allocation. (p. 281). New York: McGraw.

Sadat, A., Safari, H., Sadabadi, A. A., \& Khanmohammadi, E. (2016). An application of logarithmic fuzzy preference programming-based AHP and FRS techniques to develop and prioritize strategic objectives. Iranian Journal of Management Studies, 9(1), 149-174.

Safari, H., Faraji, Z., \& Majidian, S. (2016). Identifying and evaluating enterprise architecture risks using FMEA and fuzzy VIKOR. Journal of Intelligent Manufacturing, 27(2), 475-486.

Safari, H., \& Soufi, M. (2015). Prioritize barriers of E-factories in IRAN'S industries with hybrid multi criteria decision making (MCDM) techniques: (AHP-LLSM). Indian Journal of Fundamental and Applied Life Sciences, 5(1), 1703-1717.

Srinivasan, V., \& Shocker, A. D. (1973). Linear programming techniques for multidimensional analysis of preferences. Psychometrika, 38(3), 337-369.

Wang, J.-W., Cheng, C.-H., \& Huang, K.-C. (2009). Fuzzy hierarchical TOPSIS for supplier selection. Applied Soft Computing, 9(1), 377-386. 
Xia, H.-C., Li, D.-F., Zhou, J.-Y., \& Wang, J.-M. (2006). Fuzzy LINMAP method for multiattribute decision making under fuzzy environments. Journal of Computer and System Sciences, 72(4), 741-759.

Zhang, C.-Q., \& Lu, Y.-C. (2006). The reverse logistics evaluation based on KPCA-LINMAP model. Paper presented at the Machine Learning and Cybernetics, 2006 International Conference on.

Zheng, G., Zhu, N., Tian, Z., Chen, Y., \& Sun, B. (2012). Application of a trapezoidal fuzzy AHP method for work safety evaluation and early warning rating of hot and humid environments. Safety Science, 50(2), 228-239.
Zopounidis, C., \& Pardalos, P. M. (2010). Handbook of multicriteria analysis (1st ed.). Springer Verlag.

How to cite this article: Kashef M, Safari H, Maleki M, CruzMachado V. Solving MCDM problems based on combination of PACMAN and LINMAP. J Multi-Crit Decis Anal. 2018;25:

169-176. https://doi.org/10.1002/mcda.1650 